\title{
Submillimetre point sources from the Archeops experiment: very cold clumps in the Galactic plane ${ }^{\star}$
}

\author{
F.-X. Désert ${ }^{1}$, J. F. Macías-Pérez ${ }^{2}$, F. Mayet ${ }^{2}$, G. Giardino ${ }^{7}$, C. Renault ${ }^{2}$, \\ J. Aumont ${ }^{2}$, A. Benoît ${ }^{3}$, J.-Ph. Bernard ${ }^{4}$, N. Ponthieu ${ }^{5}$, and M. Tristram ${ }^{6}$ \\ 1 Laboratoire d'Astrophysique, Obs. de Grenoble, BP 53, 38041 Grenoble Cedex 9, France \\ e-mail: desert@obs.ujf-grenoble.fr \\ 2 LPSC, Université Joseph Fourier Grenoble 1, CNRS/IN2P3, Institut National Polytechnique de Grenoble, 53 avenue des Martyrs, \\ 38026 Grenoble Cedex, France \\ 3 Centre de Recherche sur les Très Basses Températures, BP 166, 38042 Grenoble Cedex 9, France \\ ${ }^{4}$ Centre d'Étude Spatiale des Rayonnements, BP 4346, 31028 Toulouse Cedex 4, France \\ 5 Institut d'Astrophysique Spatiale, Bât. 121, Université Paris XI, 91405 Orsay Cedex, France \\ ${ }^{6}$ Laboratoire de l'Accélérateur Linéaire, BP 34, Campus Orsay, 91898 Orsay Cedex, France \\ 7 ESA - Research and Science Support Department, ESTEC, Postbus 299, 2200 AG Noordwijk, The Netherlands
}

Received 18 September 2007 / Accepted 16 January 2008

\begin{abstract}
Aims. Archeops is a balloon-borne experiment, mainly designed to measure the Cosmic Microwave Background (CMB) temperature anisotropies at high angular resolution $(\sim 12 \mathrm{arcmin})$. By-products of the mission are shallow sensitivity maps over a large fraction of the sky (about 30\%) in the millimetre and submillimetre range at 143, 217, 353 and $545 \mathrm{GHz}$. From these maps, we produce a catalog of bright submillimetre point sources.

Methods. We present in this paper the processing and analysis of the Archeops point sources. Redundancy across detectors is the key factor allowing us to distinguish glitches from genuine point sources in the 20 independent maps.

Results. We look at the properties of the most reliable point sources, totalling 304. Fluxes range from 1 to $10000 \mathrm{Jy}$ (at the frequencies covering 143 to $545 \mathrm{GHz}$ ). All sources are either planets (2) or of galactic origin. The longitude range is from 75 to 198 degrees. Some of the sources are associated with the well-known Lynds Nebulae and HII compact regions in the galactic plane. A large fraction of the sources have an IRAS counterpart. Except for Jupiter, Saturn, the Crab and Cas A, all sources show a dust-emission-like modified blackbody emission spectrum. Temperatures cover a range from 7 to $27 \mathrm{~K}$. For the coldest sources $(T<10 \mathrm{~K})$, a steep $v^{\beta}$ emissivity law is found with a surprising $\beta \sim 3$ to 4 . An inverse relationship between $T$ and $\beta$ is observed. The number density of sources at $353 \mathrm{GHz}$ with flux brighter than $100 \mathrm{Jy}$ is of the order of 1 per degree of Galactic longitude. These sources will provide a strong check for the calibration of the Planck HFI focal plane geometry as a complement to planets. These very cold sources observed by Archeops should be prime targets for mapping observations by the Akari and Herschel space missions and ground-based observatories.
\end{abstract}

Key words. ISM: general - ISM: clouds - methods: data analysis - cosmology: observations - submillimeter - catalogs

\section{Introduction}

ARCHEOPS is a balloon-borne experiment following on from the PLANCK satellite and its High Frequency Instrument (HFI). It measures the Cosmic Microwave Background (CMB) temperature anisotropies at high angular resolution $(\sim 12$ arcmin) over a large fraction of the sky (around 30\%) in the millimetre and submillimetre range at 143, 217, 353 and $545 \mathrm{GHz}$. The main results of cosmological nature have been discussed elsewhere (Benoît et al. 2003a,b; Tristram et al. 2005). But because we have, for the first time, a large survey of the millimetre sky, studies on other scientific topics can be performed. Detection of large-scale polarized dust emission is reported in Benoît et al. (2004), Ponthieu et al. (2005). The large scale spectral properties of the dust emission have been investigated by

* Tables 3 and 4 are only available in electronic form at the CDS via anonymous ftp to cdsarc.u-strasbg.fr (130.79.128.5) or via http://cdsweb.u-strasbg.fr/cgi-bin/qcat?J/A+A/481/411
Bernard (2004) and the statistical detection of clusters of galaxies is shown by Hernández-Monteagudo et al. (2005).

Here, we analyze another by-product of the ARCHEOPS mission. We look at the properties of the most reliable point sources in the ARCHEOPS survey. We discuss the extraction method, the catalog of candidate cold dust clumps of likely galactic origin, as well as two planets and two supernova remnants. Some of these clumps are producing massive stars. Implications for the galactic clump mass distribution function and the expected number of Galactic point sources in the PLANCK survey are then discussed.

\section{Point-source extraction}

\subsection{The instrument}

The gondola contains a primary mirror with an effective $1.5 \mathrm{~m}$ diameter, a secondary mirror and a photometer containing spider-web bolometers cooled to $100 \mathrm{mK}$. The instrument is described in detail by Benoît et al. (2002) and Macías-Pérez et al. (2007). The ARCHEOPS $353 \mathrm{GHz}$ channel consists of three pairs 
of bolometers mounted on polarizer dichroics so as to detect the polarized diffuse emission of Galactic dust. The telescope boresight angle is $48 \mathrm{deg}$ with respect to the zenith. The gondola is made spins at $2 \mathrm{rpm}$ via a motor fixed on the balloon chain. The Eastward balloon trajectory and the Earth rotation make the instantaneous circle drift on the celestial sphere. This scanning strategy produces a shallow survey of a large fraction of the sky in few hours. The angular resolution varies from 15 to 8 arcmin (FWHM) with the channel frequency from 143 to $545 \mathrm{GHz}$. The sensitivities and main data processing methods are described by Macías-Pérez et al. (2007). We use the data from 6 detectors at $143 \mathrm{GHz}, 7$ at $217 \mathrm{GHz}, 6$ at $353 \mathrm{GHz}$ and one at $545 \mathrm{GHz}$.

\subsection{The method}

Because of the scanning strategy, one detector will sweep rapidly a given diffraction spot of the sky. Instead of using the timeline signature of point sources (impulse convolved with beam response) which can be confused with glitches (impulse only), we prefer to use the redundancy between the 20 available bolometers at the map level. Glitches will fall at random locations, whereas point sources will produce a concentration of bright spots in the same sky position in several bolometer maps. The detection process is a separate step from the measurement process. Once a candidate location is found, the point-source flux and error are measured on all bolometer maps and coadded with natural weights.

The data from the last flight (KS3, 2002, Feb. 7th) only are used, with a time range between 15.4 and 27.3 UT (well within night time). What is projected on sky maps is between 15.5 and 27 UT.

\subsection{Timeline processing}

The Galactic timeline processing is described by Macías-Pérez et al. (2007) and is slightly different from the CMB one. An example is shown in Fig. 1. The low frequency thermal drifts, the atmospheric emission and the Galactic diffuse emission signal produce a varying background signal. Stripes can be produced that degrade the efficiency of point-source detection. Therefore, it is necessary to subtract the baseline. The timeline, which is masked and interpolated around glitches or strong point sources, is smoothed in order to provide this baseline, to be subtracted from the initial time ordered information (hereafter TOI). The smoothing occurs, first over a period of several revolutions, then over lengths of ninety degrees on the instantaneous circle described by the gondola. Atmospheric noise can leave residues on ten degree scales. A timeline component separation is done on these scales or larger. The clean timeline (CToi) is ready for map making. We also produce a clean deglitched timeline (CDToi) to be used for faint point source and background map computations.

\subsection{Map-making}

The prepared timelines have white noise properties. Thus we proceed with a simple map-making with a natural weighting of the prepared timelines (CToi and CDToi) for each detector, using pointing information from the star sensor and the known detector position in the focal plane. The map is made in the Healpix scheme (Gorski et al. 2005) with $N_{\text {side }}=512$. It provides a 7-arcmin pixel size which is adequate to sample the point-spread-function. The map is smoothed with a 12 -arcmin
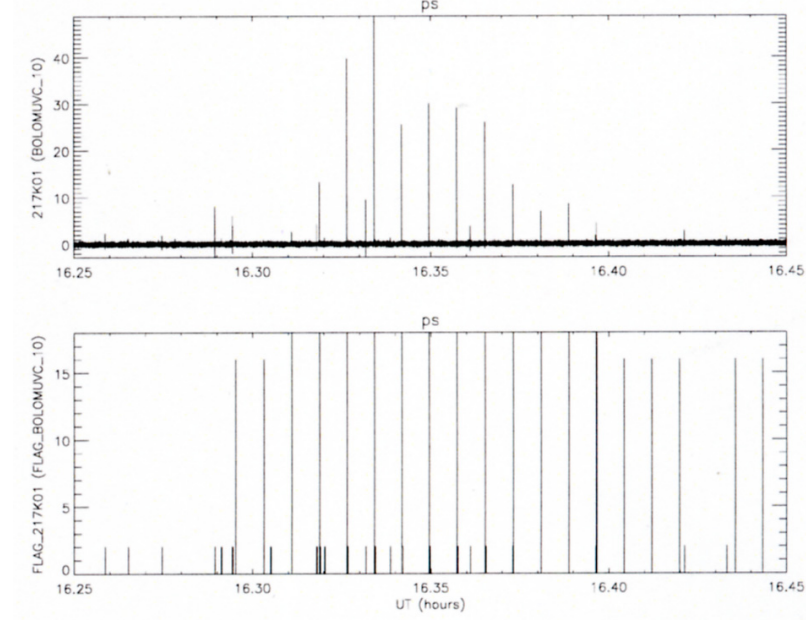

Fig. 1. Extract of the bolometer 217K01 signal timeline (upper plot). Note the regular spikes produced by Jupiter, when the gondola crosses its line of sight at each revolution (pendulation explains why the spike intensity is not smooth during the different crossings). On the other hand, cosmic ray hits happen at random times. The lower plot indicates the cumulated flagging found during data processing (a value of 2 for glitches and a value of 16 around known point sources).

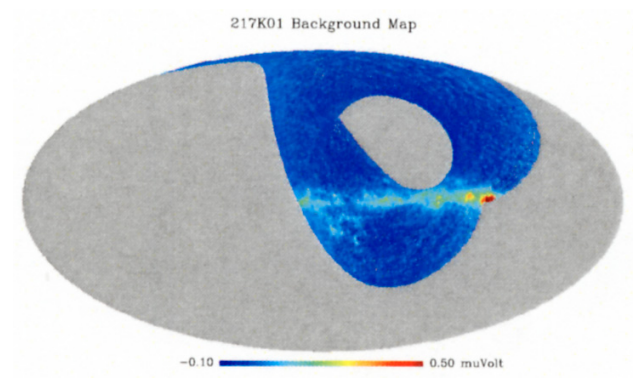

Fig. 2. Background map of the $217 \mathrm{~K} 01$ detector. This map is obtained by projecting the clean deglitched timeline and by smoothing the result with a 60 arcmin circular Gaussian kernel. An all-sky Mollweide projection, with the Galactic Anticenter at the center of the projection, is used throughout this paper. About 30 percent of the sky was observed. This smooth map is subtracted from the detector map in order to find the point sources by a simple thresholding algorithm.

circular Gaussian kernel in order to prepare for the point-source detection processing. A background component is evident with angular scales of a few degrees, due to the Galaxy diffuse component emission (mostly due to interstellar dust at these frequencies). We compute a smooth background component (Fig. 2) by projecting the CDToi and then smoothing the resulting map with a 60 arcmin circular Gaussian kernel. The smoothing is done in the spherical harmonic space. Pixels that were unobserved (at the edge of the survey) are replaced by zero before the spherical harmonic transform is done. The final backgroundsubtracted map is then computed for each detector. Thus, the end map represents for each bolometer the sky convolved with a 12-arcmin. Gaussian kernel minus a 60-arcmin. Gaussian kernel. This spatial filter has a zero mean and is appropriate for a simple point-source detection-by-thresholding algorithm. We also produce channel maps obtained by optimal coaddition of those previous maps for detectors of a given channel (i.e. at the same frequency). A relative calibration between detectors is used for that coaddition, which is described in Macías-Pérez et al. (2007). 

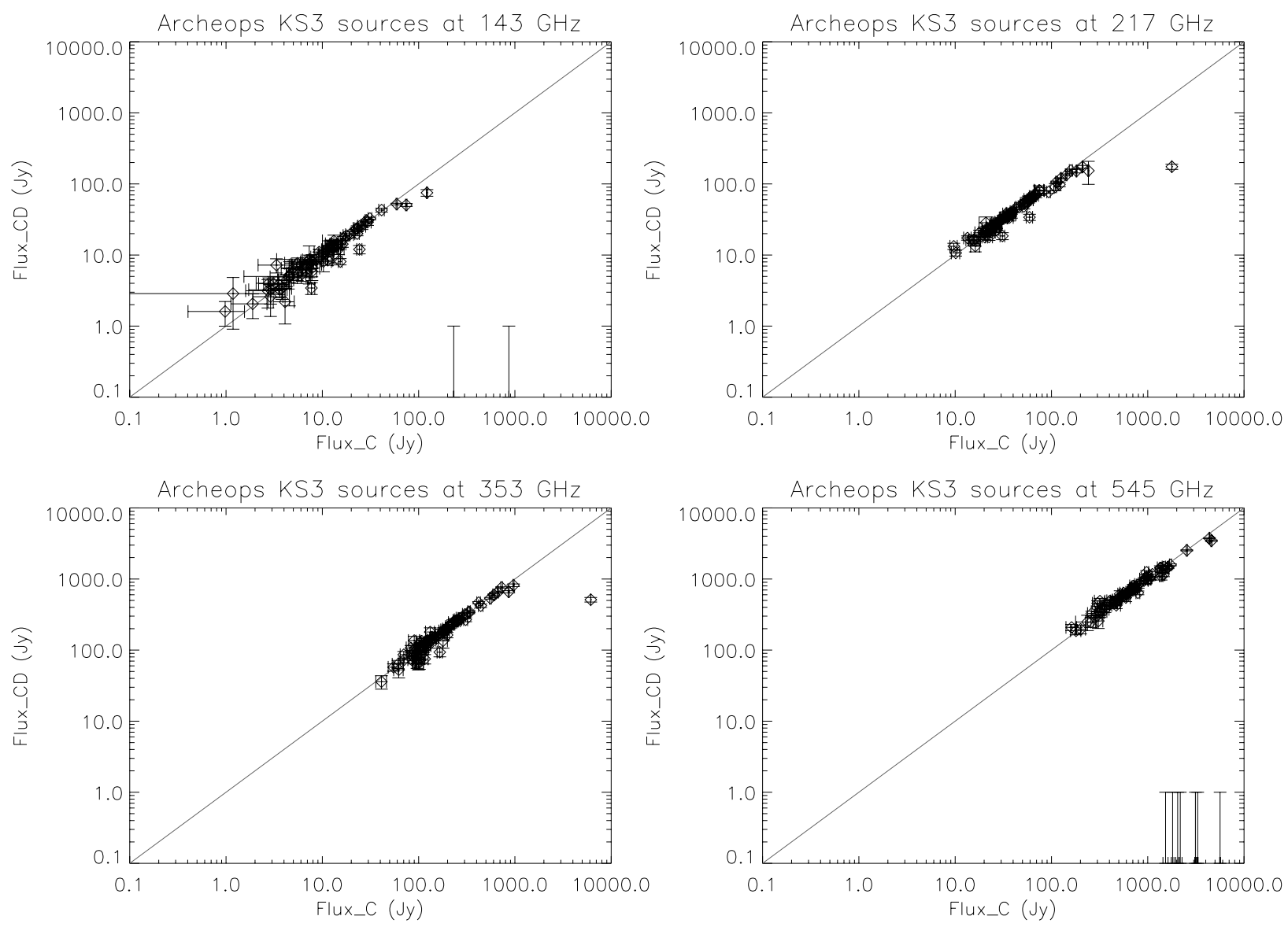

Fig. 3. Flux comparison between the two detection methods at the four frequencies, for sources in common to both methods. The plots compare the flux obtained with (CD) and without (C) a deglitching applied to the data.

\subsection{Point-source extraction}

Outside the galactic plane, the detector map is dominated by noise. A Gaussian function is fit to the histogram of the product of the pixel value $v_{\mathrm{p}}$ by the square root of the number of hits $N_{\mathrm{p}}$. For a given detector, the dispersion $\sigma_{\mathrm{d}}$ gives the typical elementary noise value for one hit, i.e. for one data sample, the data acquisition rate being $153 \mathrm{~Hz}$. It is then used to estimate the noise on each pixel as $\sigma_{\mathrm{p}}=\sigma_{\mathrm{d}} / \sqrt{N_{\mathrm{p}}}$. A listing of target pixels defined as $\frac{S}{N}=\left|\frac{v_{\mathrm{p}}}{\sigma_{\mathrm{p}}}\right|>5$ is produced for each detector. At this stage glitches and point sources are not separated. Two catalogs are obtained depending on whether one uses the CToi (still containing hits from strong point sources and glitches) or the CDToi (having strong point sources and glitches removed). For the CToi catalog, we perform a final separation of point sources from glitches by requesting that, for a point-source, the above $5 \sigma$ detection criteria for a given sky pixel be matched by at least 5 different detectors. For the CDToi catalog, we use the final point-source criterium that at least two channel maps have $4 \sigma$ detections. A channel map is defined here as the optimal average of the maps of all detectors at the same frequency. Out of the 4500 pixels that satisfy the criteria, some are connected to each other and correspond to the same point-source. To have a single position for each point-source, we keep the pixel for which a maximum number of detectors have a $5 \sigma$ detection. This defines a final catalog of sources. For each of them, we can measure the flux, error and position on the 20 independent detector maps and the 4 channel maps.
Figure 3 shows the flux comparison of the two methods (CToi and CDToi) for the 87 sources in common in the two catalogs. A very good agreement is obtained for most of the flux range and for all four frequencies. At the bright end, we expect and observe that the deglitching slightly biases the flux measured from the CDToi ("CD" fluxes in the abscissae of the figure). However, the CDToi method is more powerful at the faint end, because the signal to noise ratio benefits from the coaddition. We have therefore merged the two catalogs. For each of these candidates, we average the flux from different bolometers in each of the 4 frequency bands with a natural weight (equal to the inverse square of the noise). Positions are measured with the same weighting. From the internal dispersion between channel positions, we have estimated the $1 \sigma$ position accuracy to be about 4 arcmin (a third of the beam width). For 23 of the brightest point sources, one of the channel position disagrees with the final position. Nearby glitches in the CToi objects and confusion might cause such a systematic effect.

\subsection{Flux calibration and planet observations}

The flux calibration is performed at this stage. The CMB dipole calibration is used at 143 and $217 \mathrm{GHz}$ and the FIRAS galactic calibration is used at 353 and $545 \mathrm{GHz}$ (see details in Macías-Pérez et al. 2007). These are extended source calibrations. In order to propagate them to point-source calibration, we integrate the beams measured on Jupiter and Saturn. Assuming angular diameters for these (unresolved) sources of 44.60 by 
Table 1. Jupiter and Saturn brightness temperature, as calibrated with Archeops extended source calibrators (the CMB dipole at 143 and $217 \mathrm{GHz}$ and the Firas Galaxy at 353 and $545 \mathrm{GHz}$ ). Error bars are absolute calibration errors.

\begin{tabular}{ccccc}
\hline \hline & \multicolumn{2}{c}{ Jupiter } & \multicolumn{2}{c}{ Saturn } \\
$v(\mathrm{GHz})$ & $T_{\mathrm{RJ}}(\mathrm{K})$ & $\sigma(\mathrm{K})$ & $T_{\mathrm{RJ}}(\mathrm{K})$ & $\sigma(\mathrm{K})$ \\
\hline $\mathbf{1 4 3}$ & 165 & 20 & 160 & 19 \\
$\mathbf{2 1 7}$ & 139 & 22 & 144 & 23 \\
$\mathbf{3 5 3}$ & 159 & 24 & 179 & 27 \\
$\mathbf{5 4 5}$ & 146 & 20 & 166 & 23 \\
\hline
\end{tabular}

41.71 arcsec and 19.05 by 17.00 arcsec respectively, we obtain the brightness temperature given in Table 1. Statistical error bars are negligible. The total error bars are made up from an absolute calibration error (resp. 4, 8, 12, and 8\%), the estimated error due to the beam integration procedure (estimated by comparing the beam shape obtained from Jupiter and Saturn observations at the same frequency) and an intercalibration error (the measurement dispersion across the bolometers at the same frequency).

Figure 4 shows the (sub)millimetre spectrum of Jupiter and Saturn, as measured by WMAP (Page et al. 2003), ARCHEOPS and Goldin et al. (1997). There is a broad agreement of the flux scale over a factor of 20 in frequency range. The ratio of Jupiter to Saturn flux at a given frequency, which should be independent of the absolute flux scale calibration, is larger in ARCHEOPS than in the Goldin et al. (1997) measurements. Explanations might be sought in small non-linearity problems in the ARCHEOPS instrument for these very high flux sources and also from the simplified emission assumptions of the two planets (inclination of Saturn rings), as shown by the detailed PRONAOs calibration (Pajot et al. 2006).

In addition to the systematic errors discussed above, for the point source catalog, we need to consider the error introduced by the flux measurement method described in Sect. 2.5. The convolution by a simplified 2D Gaussian kernel increases the measurement intrinsic dispersion of bolometers at a single frequency. For the final catalog, we estimate the absolute calibration scale uncertainties as $14,21,17$, and $15 \%$ at $143,217,353$, and $545 \mathrm{GHz}$ respectively.

\section{Results}

\subsection{The point-source catalog}

ARCHEOPS has detected 304 submillimetre point-sources in the covered $30.0 \%$ fraction of the sky. These are plotted in Fig. 5. They are mostly in the Galactic Plane, with a high concentration in the Cygnus and Taurus complexes. Fluxes range from 1 to $10^{4} \mathrm{Jy}$ and median average fluxes and errors are 3.0 and $1.4 \mathrm{Jy}$ at $143 \mathrm{GHz}, 19$ and $3 \mathrm{Jy}$ at $217 \mathrm{GHz}, 76$ and $13 \mathrm{Jy}$ at $353 \mathrm{GHz}$, and 344 and $44 \mathrm{Jy}$ at $545 \mathrm{GHz}$. The survey is inhomogeneous in sensitivity because the scanning strategy and the limited observing time did not allow an equal number of pixel hits everywhere. The obtained median sensitivities are in agreement with expectations from Table 8 by Macías-Pérez et al. (2007), giving us some confidence in the processing efficiency. The average integration time spent by an ARCHEOPS detector on a 12-arcmin spot on the sky is only $0.11 \mathrm{~s}$.

The catalog of sources (the 2 planets being excluded) is given in Table 3 . It includes the position on the sky with galactic coordinates in degrees, fluxes in Jy, with their error bars and signal to noise ratio. Associations with the IRAS point-source and small extended source catalog are given in Table 4. The
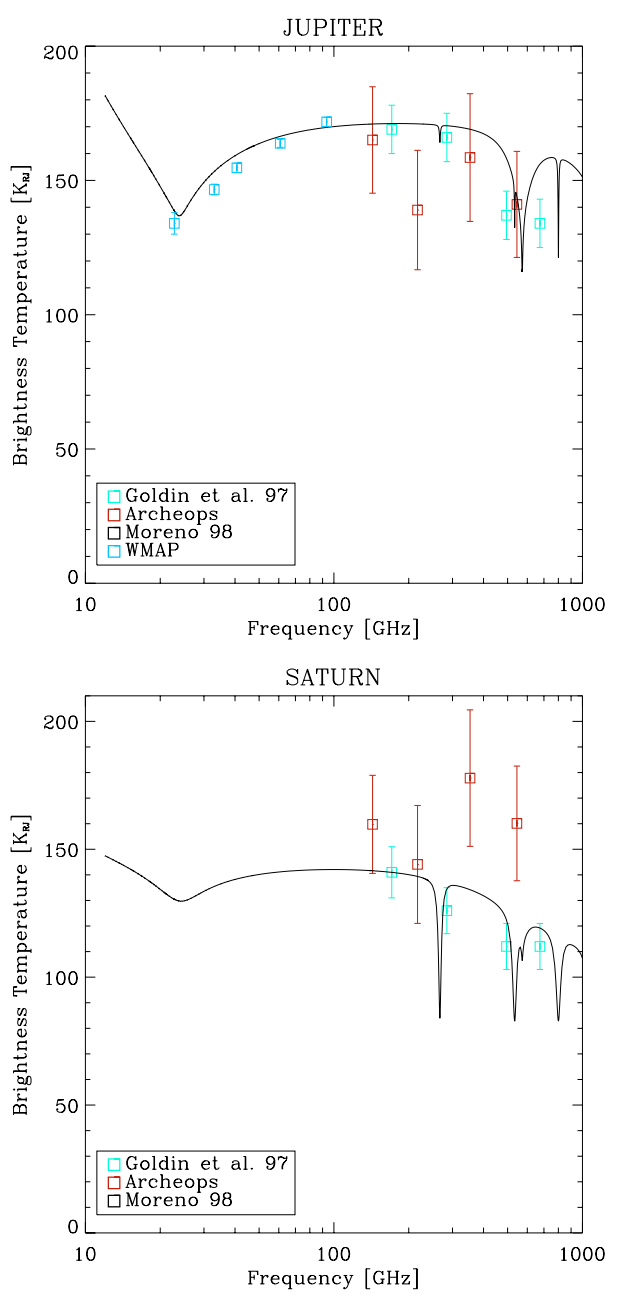

Fig. 4. Jupiter (upper) and Saturn (lower) flux measurements (squares) compared with Moreno's atmosphere models (lines) (Moreno 1998) as well as WMAP and Goldin et al. (1997)'s measurements.

matching radius is 10 arcmin ( $2.5 \sigma$ of ARCHEOPS position accuracy). In many cases, there is more than one IRAS counterpart within the matching radius, so the source with the strongest flux at $100 \mu \mathrm{m}$ (IRAS) was selected. Associations with previously known sources are made with CDS catalogs ${ }^{1}$ (Table 4). Many sources have a counterpart with a bright or dark Lynds Nebula or an HII compact (Sharpless) region (for example, DR 21, W 3). Matches to HII regions with an angular size lower than 10 arcmin from the catalogue of Paladini et al. (2003) are also included. Finally, in Table 4, sources whose IRAS counterpart is likely an ultra-compact HII region (UCHII) are indicated. These were identified from their IRAS color following the color criteria from Kurtz et al. (1994) and applying a flux threshold of $100 \mathrm{Jy}$ at $100 \mu \mathrm{m}$ (i.e. only sources with the colors of an ultra-compact HII region and $F(100 \mu \mathrm{m})>100 \mathrm{Jy}$ are indicated).

Concentration of the sources in the Galactic Plane and in molecular cloud regions indicates a galactic origin. This galactic origin of most of the sources is confirmed in Fig. 6. We compute the background expected from IRAS extrapolations (Finkbeiner et al. 1999) at the same frequency as the ARCHEOPS sources. There is not a direct correlation between the ARCHEOPS flux at $353 \mathrm{GHz}$ and the diffuse background emission expected at the same frequency, but we find in Fig. 6 that the sources tend to gather in high background regions. For example, $60 \%$ of the

1 http://cdsweb.u-strasbg.fr 


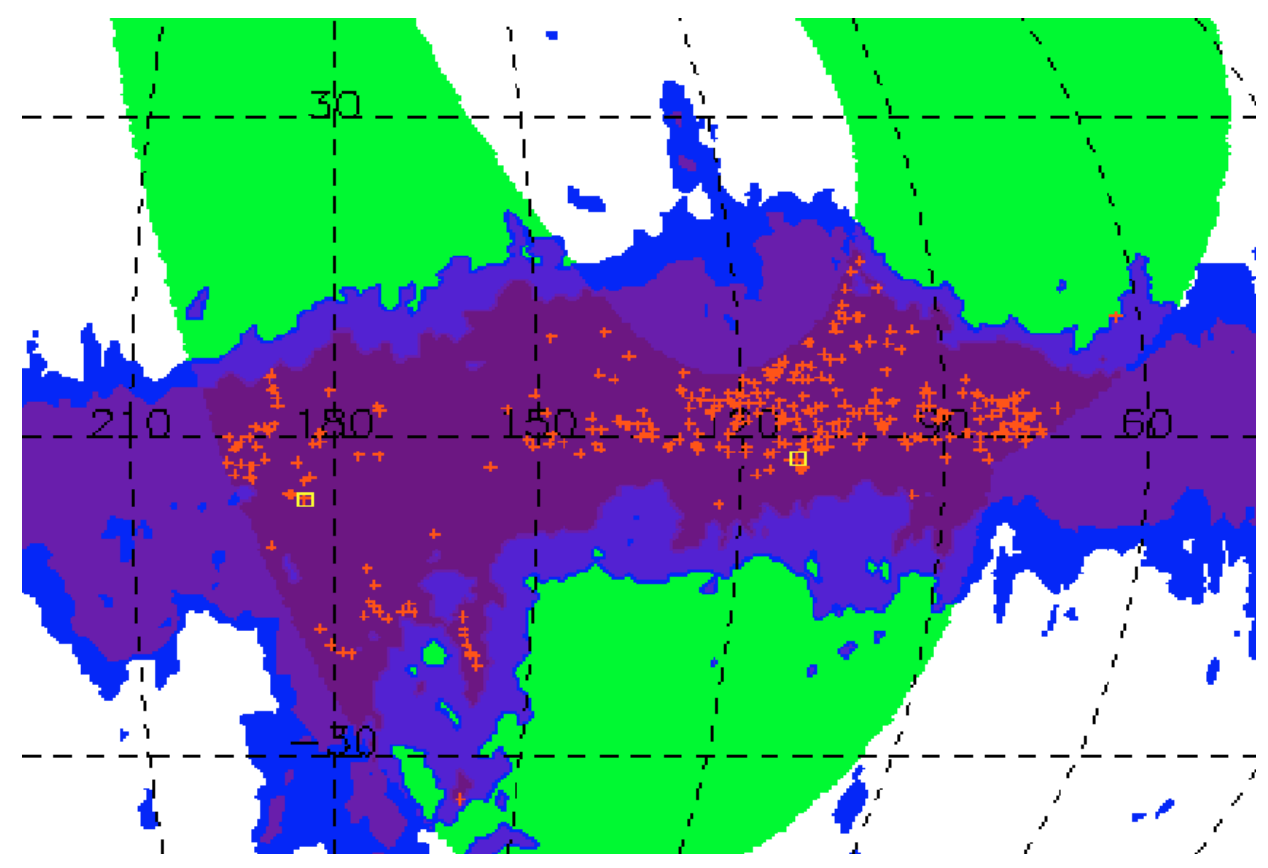

Fig. 5. Location of the point sources (in red pluses) in galactic coordinates in a Mollweide projection. The Crab (Tau A) and Cas A correspond to the yellow squares (from left to right). Most of the sources are concentrated in the Galactic plane. The ARCHEOPS survey is shown as the green area. A thresholded IRAS galactic map (blue) aids to trace high far-infrared background regions.

Table 2. Submillimetre fluxes and the statistical flux errors of the two supernova remnants detected by ARCHEOPS.

\begin{tabular}{ccccc}
\hline \hline & \multicolumn{2}{c}{ Cas A } & \multicolumn{2}{c}{ Crab } \\
$v(\mathrm{GHz})$ & $F(\mathrm{Jy})$ & $\sigma(\mathrm{Jy})$ & $F(\mathrm{Jy})$ & $\sigma(\mathrm{Jy})$ \\
\hline $\mathbf{1 4 3}$ & 74.5 & 1.3 & 231.4 & 1.4 \\
$\mathbf{2 1 7}$ & 58.3 & 2.9 & 181.9 & 1.8 \\
$\mathbf{3 5 3}$ & 121 & 14 & 185 & 11 \\
$\mathbf{5 4 5}$ & 359 & 36 & 236 & 58 \\
\hline
\end{tabular}

point sources are in regions with an average brightness larger than $5 \mathrm{MJy} / \mathrm{sr}$ at $353 \mathrm{GHz}$. Similarly, using the large scale Galactic CO survey by Dame et al. (2001), we do not find a direct correlation between the $\mathrm{CO}$ velocity-integrated brightness and the ARCHEOPS flux but there is a strong trend for the submillimetre sources to lie in high $\mathrm{CO}$ background regions. For example, half the point sources lie on top of a CO background greater than $15 \mathrm{~K} \mathrm{~km} \mathrm{~s}^{-1}$ whereas half of the pixels recorded by ARCHEOPS with a CO measurement have a CO flux greater than only $2.5 \mathrm{~K} \mathrm{~km} \mathrm{~s}^{-1}$

\subsection{Photometry}

We have compared the fluxes measured with ARCHEOPS with previous surveys conducted in the submillimetre domain.

In particular the Crab and Cas A point-sources are wellknown sources and can be used for comparison. No other supernova remnant (Green 2004) could be associated with the present catalog.

Concerning the Cas A supernova remnant, ARCHEOPS confirms the submillimetre emission (Table 2) in excess of the synchrotron component and discovered and mapped by SCUBA (Dunne et al. 2003). The $143 \mathrm{GHz}$ point closely fits the synchrotron extrapolation (giving some confidence in the ARCHEOPS photometric calibration), whereas a large excess exists at 217, 353, and $545 \mathrm{GHz}$. The origin of

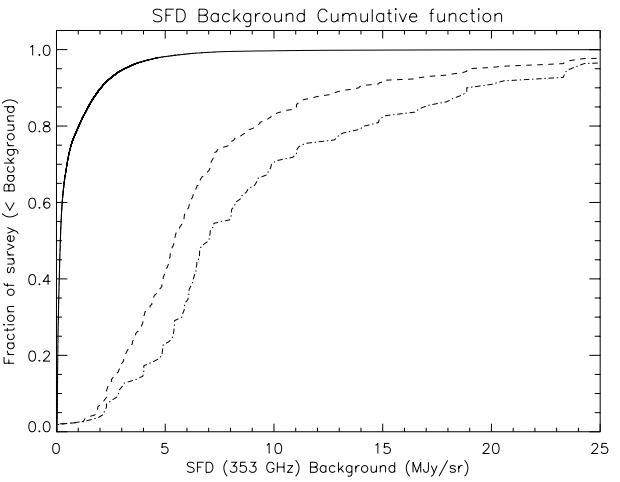

Fig. 6. Cumulated fraction (dashed curve) of sources falling below a given background. Here, sources are counted at the $353 \mathrm{GHz}$ frequency. The background is measured as pixel value of a $353 \mathrm{GHz}$ SFD map, smoothed with a 30 arcmin wide kernel. The dot-dash line shows the cumulated fraction of ARCHEOPS point sources with a flux larger than $100 \mathrm{Jy}$. The black curve shows the cumulated fraction of all pixels visited by ARCHEOPS.

this excess (cold dust, iron needles) has been debated by Dwek (2004) and Gomez et al. (2005) and is studied by Macías-Pérez et al. (2008b).

The Crab photometry is further analyzed in Macías-Pérez et al. (2008a). The $143 \mathrm{GHz}$ measurement is in agreement with the expected radio synchrotron component described by a power law with a spectral index $\beta \simeq-0.299 \pm 0.009$ (Baars et al. 1977).

There is not a single common source with the WMAP cata$\log$ (Hinshaw et al. 2007), because the WMAP catalog contains only extragalactic radio sources.

Sources observed by large ground-based telescopes are much fainter than detected as a point-source by the ARCHEOPS balloon experiment. We find that ARCHEOPS fluxes are usually greater than some of the ground-based measurements on 

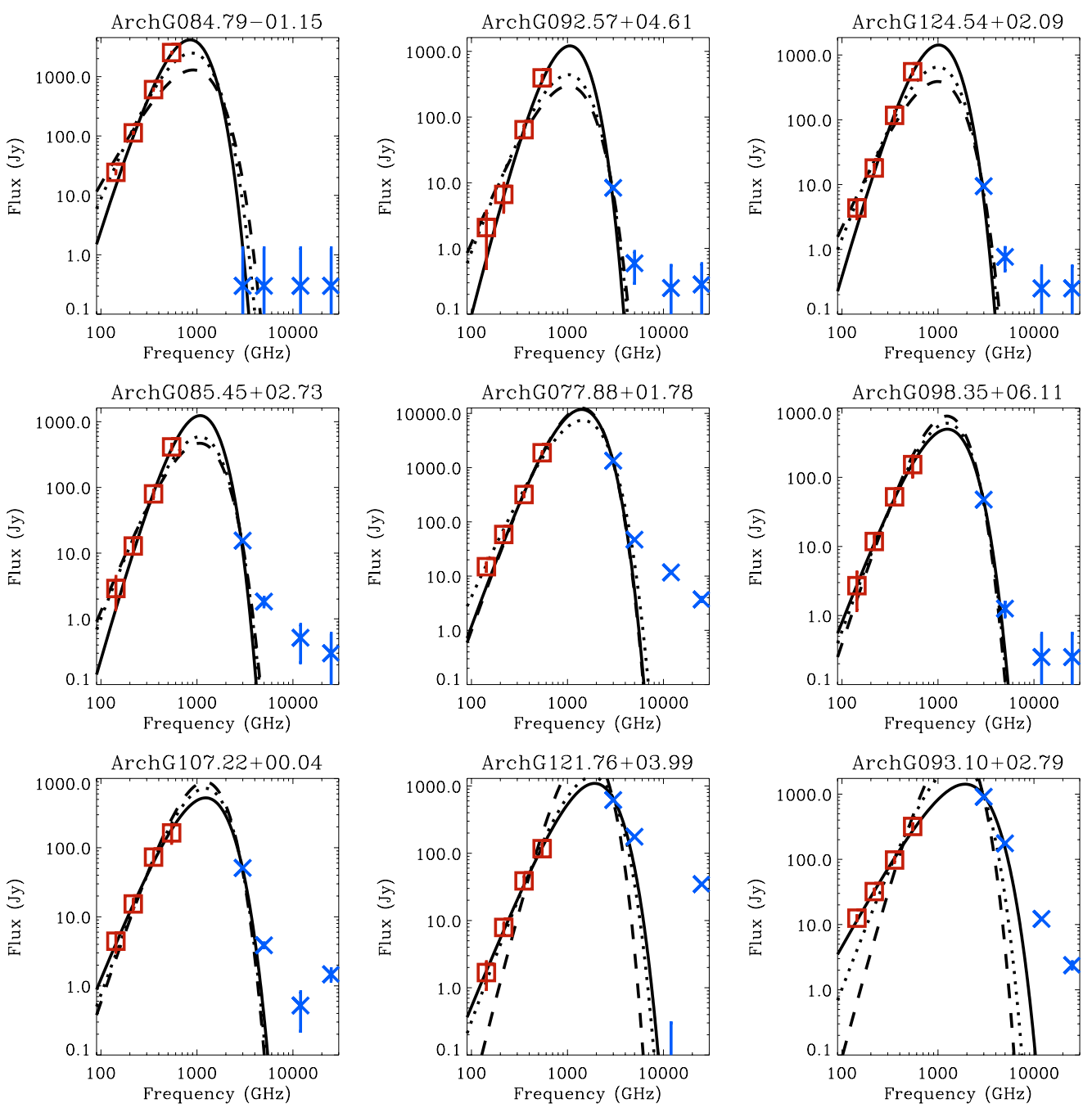

Fig. 7. Examples of millimetre and far infrared spectra of ARCHEOPS sources. ARCHEOPS measurements are shown with error bars as the red squares. IRAS measurements (from the point-source catalog or the small extended source catalog if present, Beichman et al. 1988) are noted as blue crosses with their error bars. A modified blackbody law fit to the ARCHEOPS and the $100 \mu \mathrm{m}$ IRAS flux is shown as a solid line. The dotted curve is obtained by fixing the emissivity exponent at a value of 2 . The dashed curve is obtained by fixing the temperature at $11 \mathrm{~K}$. Note the steepness of the spectrum of very cold sources in the three upper plots (sources ArchG084.79-01.15, ArchG092.57+04.61, ArchG124.54+02.09) with respect to the last lower plot on the right (source ArchG093.10+02.79).

integrated mapped regions. However, most of the present sources have never been measured at these frequencies and spatial resolution before. We can compare the flux values with the few available values $(\sim 10)$ published by Chini et al. (1984), Chini et al. (1986a), Chini et al. (1986b), and measured with a 90 arcsec beam ground-based photometer at $230 \mathrm{GHz}$. ARCHEOPS values are always above the ground-based observations by a factor that can reach 10 . We think that the photometric disagreement is due to the chopping techniques used in groundbased experiments and to the spatial extent of the sources.

No extragalactic point-source can be identified ${ }^{2}$ in the present catalog. The strongest known extragalactic point source (for a 12-arcmin beam) is M 82 which has a flux of about $5 \mathrm{Jy}$ at $353 \mathrm{GHz}$. It is not in our survey coverage, and photometrically, it is below our sensitivity limit.

\footnotetext{
2 We measured the Andromeda (M 31) extended source flux within IRAS $100 \mu \mathrm{m}$ contour of $5 \mathrm{MJy} / \mathrm{sr}$ (about one degree size), and found a marginal detection $(3 \sigma)$ at $545 \mathrm{GHz}$ only: fluxes of M 31: $4407 \mathrm{Jy}$ at IRAS $100 \mu \mathrm{m},<26 \mathrm{Jy}$ at $143 \mathrm{GHz},<90 \mathrm{Jy}$ at $217 \mathrm{GHz},<340 \mathrm{Jy}$ at $353 \mathrm{GHz}$ and $660 \pm 200 \mathrm{Jy}$ at $545 \mathrm{GHz}$. Upper limits are quoted as $2 \sigma$.
}

\subsection{Submillimetre spectra}

Beside the 4 brightest sources (two planets and two SN remnants) most of the sources have a steep spectrum rising in the submillimetre domain with frequency, with typically an increase in flux by a factor of 100 when going from $143 \mathrm{GHz}$ to $545 \mathrm{GHz}$. That excludes synchrotron or free-free emission as the main emission mechanism. Interstellar dust is most likely the source of such spectra.

Figures 7 and 8 show the millimetre and infrared spectrum of a sample of the ARCHEOPS sources along with the IRAS measurements.

We have fitted these spectra with a simple modified blackbody law:

$F_{v} \propto v^{\beta} B_{v}(T)$

with a single dust component at a temperature $T$ and an emissivity index of $\beta$. The IRAS flux at $100 \mu \mathrm{m}$ mostly determines the temperature, whereas ARCHEOPS data points lead the fit of the emissivity exponent. It is clear that the peak emission of these 

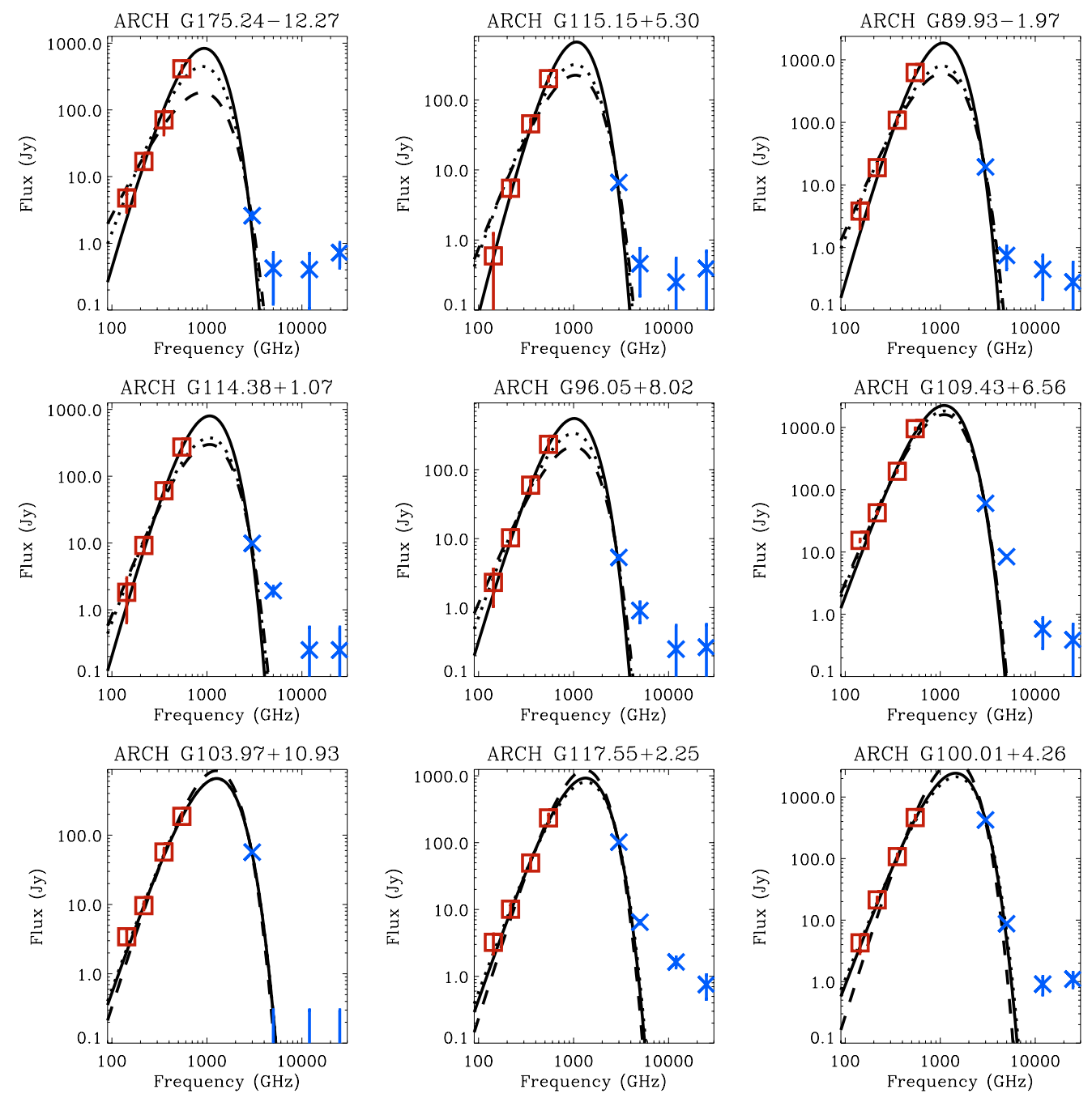

Fig. 8. More examples of millimetre and far infrared spectra of ARCHEOPS sources. ARCHEOPS measurements are shown with error bars as the red squares. IRAS measurements are noted as blue crosses. See Fig. 7 for explanations of the fitting lines.

sources happens at the $\mathrm{THz}$ frequency and will have to await HERSCHEL and PLANCK observations.

The emissivity law exponent $\beta$ and the temperature $T$ quoted in Table 4 are found by fitting the four ARCHEOPS fluxes and the $100 \mu \mathrm{m}$ IRAS flux. The fit is generally good except for $15 \%$ of the sources where the $353 \mathrm{GHz}$ flux shows a small systematic deficit, or when IRAS or some ARCHEOPS band measurements are missing. The goodness of the fits has been tested using a $\chi^{2}$ (quoted in Table 4) goodness-of-fit criteria at the 2- $\sigma$ level. In Table 4 when the fit is unsatisfactory, we quote the emissivity index $\beta_{11}$ obtained by fixing the temperature to $11 \mathrm{~K}$ in the fitting procedure, as well as the temperature $T_{2}$ obtained by fixing the emissivity exponent $\beta$ at the fiducial value of 2 (only statistical error bars are quoted). In the following we will only consider the global two-parameter ( $\beta$ and $T$ ) fit unless otherwise stated. The fiducial temperature of $11 \mathrm{~K}$ was chosen as close to an average of the temperatures found in the two-parameter fits (see also Fig. 10).

\subsection{Submillimetre point-source number counts}

Excluding the four brightest sources (Jupiter, Saturn, Crab and Cas A), the study of the submillimetre spectrum of the ARCHEOPS sources indicates that they may constitute a homogenous set of dust-emission sources. Therefore, we can compute the number count of the Archeops sources. For this purpose, we fiducially choose the $353 \mathrm{GHz}$ data. Figure 9 represents the un-normalized number counts of the $353 \mathrm{GHz}$ sources as a function of flux. We see a strong increase of the number counts with decreasing flux down to about $100 \mathrm{Jy}$, which is well above the $4 \sigma$ sensitivity level for ARCHEOPS. Then the number counts start decreasing with decreasing flux. This is mostly due to the sensitivity cutoff of Archeops at $353 \mathrm{GHz}$. At the high flux range, the number counts $F \mathrm{~d} N / \mathrm{d} F$ is consistent with a power law with an exponent of -1.5 .

The survey mostly covers the Galactic Plane between the galactic longitudes of 75 and 198 degrees. We can thus normalize the number counts to one degree of Galactic longitude. We obtain the following integral number counts:

$N\left(>F_{v}\right)=(1.0 \pm 0.1$ source $/ \mathrm{deg})\left(\frac{F_{v}(353 \mathrm{GHz})}{100 \mathrm{Jy}}\right)^{-1.5 \pm 0.2}$.

The number counts for the other ARCHEOPS frequencies can be computed by using a scaling factor of the form $100 \mathrm{Jy}(v / 353 \mathrm{GHz})^{4}$, where $v$ is the required frequency.

Assuming a constant latitude width of 10 degrees, the confusion limit, defined as one source every thirty beams, is $\sim 27 \mathrm{Jy}$. 


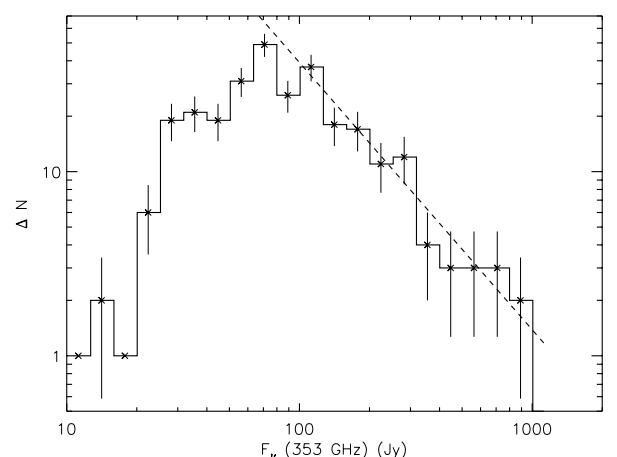

Fig. 9. Un-normalized number counts of the $353 \mathrm{GHz}$ ARcheOPS sources. Error bars in each histogram bin are assumed to follow a Poisson law. A best-fit power law with an exponent of $-1.5 \pm 0.2$ is traced as the dashed line. Incompleteness starts below 100 Jy flux.

This is well below the ARCHEOPs detection limit. The pointsource contribution to the diffuse background is:

$B_{v}\left(>F_{v}\right)=0.09 \mathrm{MJy} / \mathrm{sr}\left(\frac{F_{v}(353 \mathrm{GHz})}{100 \mathrm{Jy}}\right)^{-0.5}$,

which is a negligible fraction of the average brightness in the Galactic Plane, unless we extrapolate the number counts to sub-Jansky levels. We can thus conclude that the galactic submillimetre sky is dominated by diffuse emission and not by point-sources.

\section{Discussion}

Due to the limited angular resolution of ARCHEOPS, the observed point-sources are merely clumps of very cold interstellar matter. They are a sub-population of IRAS Galactic pointsources or slightly extended sources which are important for the study of the early stages of star formation. We have already noted the photometric disagreement between ground-based and the present balloon-borne measurements. Wings in shallow column density profile around very dense clumps might explain the larger fluxes in ARCHEOPS photometry. Also, in order to prepare the analysis of future high frequency CMB experiments like the PLANCK satellite mission, it would be important to be able to simulate these very cold clumps in the whole sky using their spectral behavior and high frequency surveys like IRAS. We now discuss their statistical properties.

\subsection{Submillimetre spectral properties}

In Fig. 10 we trace the emissivity law exponent $\beta$ as a function of temperature $T$ for the best-fit model to the spectrum of the ARCHEOPS sources. We consider only those sources for which the fit satisfies the $\chi^{2}$ goodness-of-fit criteria at 95\% C.L. We find that most of the sources have low temperatures ranging from 7 to $27 \mathrm{~K}$. The emissivity exponent increases with decreasing temperature, going up to 4 for sources below $10 \mathrm{~K}$.

The interpretation of this trend is made difficult by the intrinsic correlations between $\beta$ and $T$ parameters, as shown in Fig. 11, where the 1- $\sigma$ confidence level contour is plotted for each source. We have to assess whether the observed $\beta-T$ trend can be completely due to the natural correlation of errors. For this purpose, we parametrize the trend with a simple power-law:

$\beta=A T^{-\gamma}$.

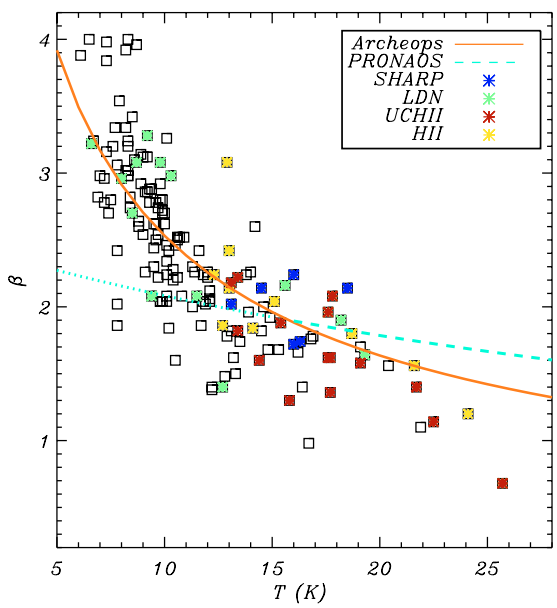

Fig. 10. Dust emissivity exponent $\beta$ vs. temperature $T$ for the ARCHEOPS sources (black squares). With red, green and blue stars we represent those ARCHEOPS sources identified as UCHII (ultracompact HII), SHARP (Sharpless HII) and LDN (Lynds Dark Nebulae) sources respectively. With the orange solid line and light blue dashed line we overplot the ARCHEOPS and PronaOs $\beta-T$ relationships as discussed in the text.

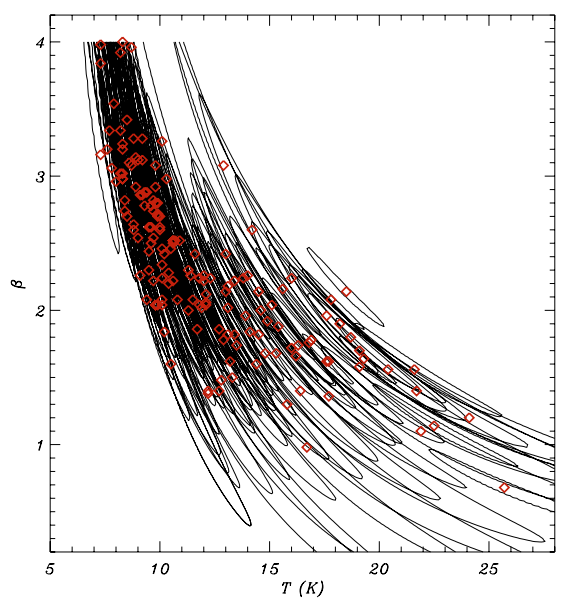

Fig. 11. Likelihood contour plot in a $\beta-T$ plot. Around each point source, a contour gives an uncertainty equivalent to a $2-\sigma$ level. Note that the correlation between the 2 parameter uncertainties makes it difficult to ascertain the true correlation between the parameters.

Figure 12 represents the $1-3 \sigma$ confidence level marginalized over $T$ for the likelihood in the $A-\gamma$ plane. A constant $\beta$ value $(\gamma=0)$ is clearly excluded at better than $99.9 \%$ C.L.

The best fit is given by the empirical law:

$\beta=(11.5 \pm 3.8) \times T^{-0.66 \pm 0.054}$,

with error bars, after marginalization, at the 0.997 C.L. The standard $\beta=2$ is obtained for $T=14.1 \mathrm{~K}$. This law is overplotted (solid line) in Fig. 10. This inverse $\beta-T$ relationship could also be biased by the selection of the IRAS counterparts to the ARCHEOPS sources. To prove that this is not the case, we have obtained the IRAs fluxes at the position of the Archeops sources by applying the ARCHEOPS map-based source extraction algorithm (see Sect. 2.5) to the IRIS maps (Miville-Deschênes \& Lagache 2005). These fluxes were then used to repeat the fit to the spectrum of each of the ARCHEOPS sources as above. The new fit leads to the same conclusions. Dupac et al. (2003) have also claimed an anticorrelation between $\beta$ and $T$ for dust sources with temperatures in the range 


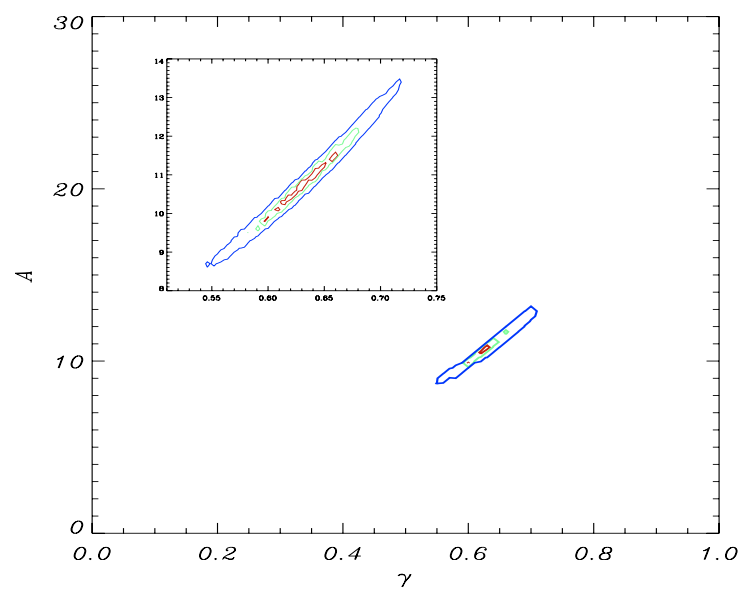

Fig. 12. Likelihood diagram in the $A-\gamma$ parameter plane (see Eq. (4)) marginalized over $T$. Contours correspond to the 0.68, 0.95, 0.9999 C.L. $\gamma=0$ is excluded by the data at more than 0.9999 C.L. indicating a real dependence between $T$ and $\beta$. Embedded plot is an expanded version of the diagram centered on the maximum of the likelihood.

$12-20 \mathrm{~K}$, as measured with the PRONAOs balloon experiment. For comparison we trace (with a dashed line, and a dotted line for the extrapolation to lower temperatures) in Fig. 10 the $\beta-T$ relationship they obtained. ARCHEOPS and PRONAOS data show a similar behavior, even though the amplitude of the variations is larger for ARCHEOPS. The inverse $\beta-T$ relationship is not explained by standard interstellar dust models and would require us to invoke specific modifications of the optical properties of the dust, such as the ones produced in amorphous disordered material, as described by Mény et al. (2007). According to such models, $\beta$ varies not only with temperature but also with wavelength. Therefore, the slight differences observed between the $\beta-T$ relationship by the two experiments could be due to ARCHEOPS observing at longer wavelengths $(500 \mu \mathrm{m}-2 \mathrm{~mm})$ than PRONAOs $(200-500 \mu \mathrm{m})$. Note that laboratory measurements of dust analogs exist that have revealed an increase of $\beta$ with wavelengths (see Boudet et al. 2005), in qualitative agreement with the observations. The observed effect is in the opposite direction to the diffuse interstellar medium colour trend, where $\beta$ is measured to be low at low temperatures (Lagache et al. 1998). To measure the absolute value of the emissivity law (which is beyond the scope of this paper) and scale it to near infrared and visible values, would require an exhaustive coherent analysis combining the submillimetre and far-infrared emission properties with the near infrared extinction properties of these clouds.

\subsection{Submillimetre spectrum compared to far infrared IRAS colours}

We check whether these very cold clumps present special features in their far-infrared IRAS colours that would make them easy to identify at high frequency. The left plot of Fig. 13 shows that the IRAS far-infrared colours of these clumps broadly lie in two regions. For large infrared fluxes, the clumps have relatively warm colours. These sources likely contain HII regions, as exemplified by the association catalog (Table 4). On the other hand, the majority of ARCHEOPS sources have far-infrared colours similar to the general galactic point-source value $(\sim 0.2)$. More quantitatively, in the right plot of Fig. 13, we show the normalized histogram of the far infrared IRAS colours for the $|b| \leq 10$ deg IRAS point-sources catalogue (light blue) with respect to the histogram of ARCHEOPS sources (red-filled). We observe that the distribution of the ARCHEOPS point-sources is similar to that of the Galactic IRAS point-sources with an excess of ARCHEOPS sources on the warm IRAS side.

We trace in the upper plot of Fig. 14, the far infrared IRAS colours for the Archeops sources as a function of temperature $T$. The far infrared IRAS colours of the ARCHEOPS sources seem to be uncorrelated with the submillimetre temperature. For most of the sources, one dust component cannot simultaneously explain the submillimetre and far-infrared colours. On the contrary, in the lower plot of Fig. 14, a single dust component is able to explain the submillimetre colours, albeit with a surprisingly large emissivity index. Therefore the far-infrared 60 to $100 \mu \mathrm{m}$ ratio is not representative of the submillimetre dust temperature. Several dust components are needed for most sources to explain the infrared and submillimetre spectra together.

From this analysis, we find that the ARCHEOPS sources show no distinct features in their far infrared IRAS colours, except for the UCHII subpopulation. This indicates that we cannot easily predict the fluxes at submillimeter frequencies of known IRAS point-sources. Only $\sim 1.3 \%$ of the IRAS sources at low galactic latitudes $\left(|b| \leq 10^{\circ}\right)$ are detected by ARCHEOPS with a flux $F_{v}(353 \mathrm{GHz}) \geq 50 \mathrm{Jy}$.

Furthermore, a significant fraction $(\sim 10 \%)$ of ARCHEOPS point-sources are not associated with any IRAS point-source making it even more difficult to estimate the point-source properties in future submillimeter surveys like PLANCK HFI.

\subsection{Ultra compact HII regions}

Forty ARCHEOPS sources are associated with ultra compact HII regions (UCHII, see Sect. 3.1). These regions are of particular physical interest as they are formed by young stars in the first steps of their evolution.

In Fig. 10 we show as red stars the best-fit temperature $T$ and dust spectral index $\beta$ for the ARCHEOPS sources associated with compact or ultra compact HII regions. We consider only those sources for which the $T-\beta$ fit satisfies the $\chi^{2}$ goodnessof-fit criteria at $95 \%$ C.L.: 23 out of 66 . Across the whole temperature range, we observe that these sources follow the general $\beta-T$ relationship described in Sect. 3.3: $\beta$ increases for decreasing $T$. However, these sources seem to be always hotter than $13 \mathrm{~K}$, likely an effect of the far-infrared selection. Note that the free-free emission does not seem to perturb the low-frequency part of the spectrum in these sources.

We also paid attention to other types of sources like dark Lynds Nebulas (LDN) and Sharpless HII regions. The bestfit temperature $T$ and dust spectral index $\beta$ for the ARCHEOPS sources associated with them are also represented in Fig. 10 as green and blue stars for the LDN and the Sharpless HII regions respectively. The ARCHEOPS point-sources associated with Sharpless HII regions tend to be hotter than $13 \mathrm{~K}$, similarly to UCHII regions. The clouds associated with LDNs seem to be preferentially at low temperatures $(T<15 \mathrm{~K})$ but they follow the general $T-\beta$ relationship.

The fact that the submillimeter properties of the HII-associated clumps are not changed (except that they are hotter) may be interpreted in the following way: the submillimetre clumps could be thought of as some precursor stage of the site for massive star production. Some of those clumps have already started producing massive stars but those massive stars have not modified the global properties of the clump enough (except through radiative heating), perhaps because they have escaped from the cloud, or because the cloud is much more 

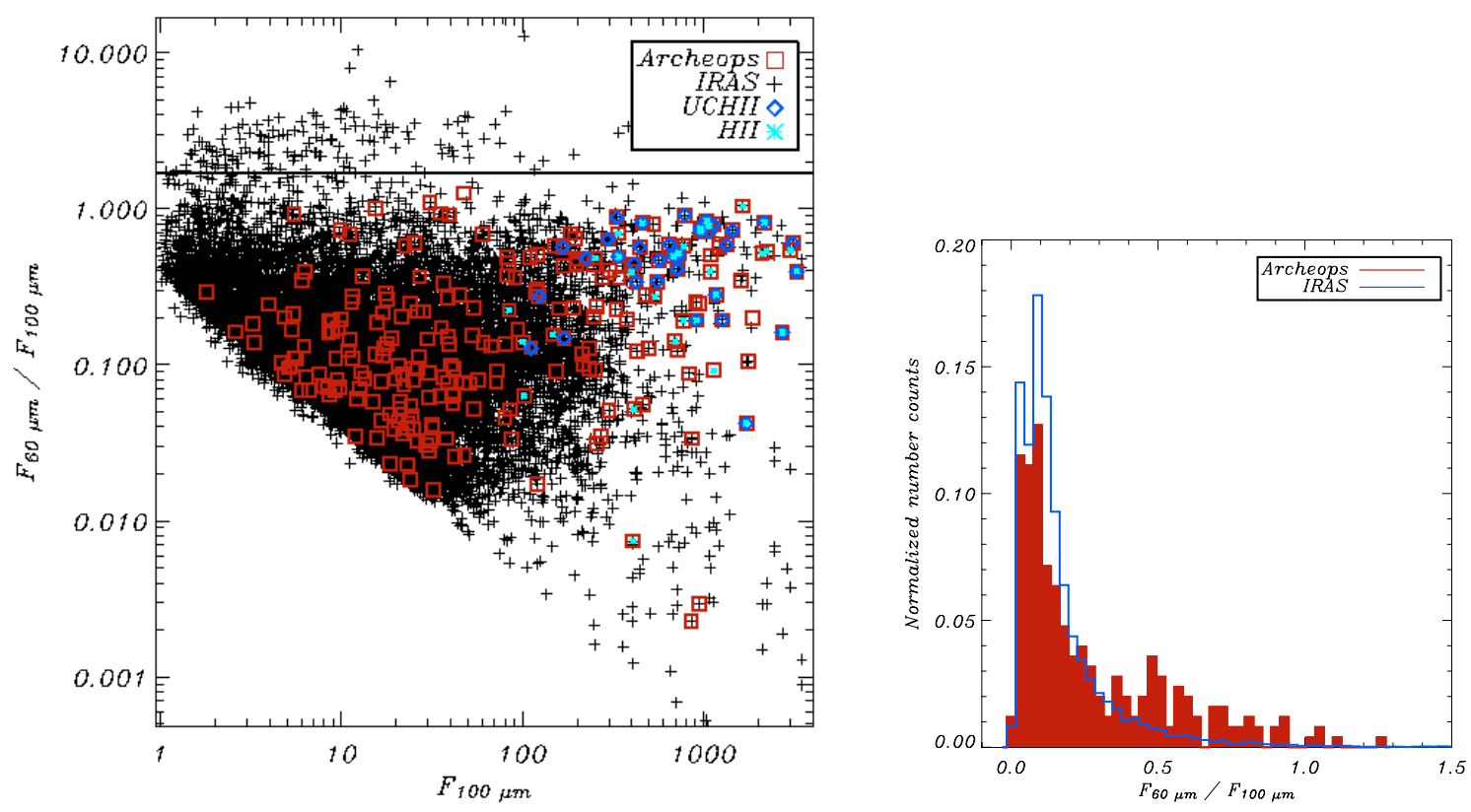

Fig. 13. Left plot: far infrared IRAS colour of point sources as a function of $100 \mu \mathrm{m}$ IRAS flux. The pluses correspond to the whole IRAS pointsource catalog at low galactic latitudes $(|b|<10 \mathrm{deg})$ with detections at both 60 and $100 \mu \mathrm{m}$. The red squares are the IRAS colours of ARCHEOPS point-sources (when detected in IRAS). HII regions are concentrated on high fluxes and warm colours. Right plot: histogram of the far infrared IRAS colour for the whole IRAS catalogue (blue) of point sources at low galactic latitudes $(|b|<10 \mathrm{deg}$ ) with detections at both 60 and $100 \mu \mathrm{m}$ and for the subsample of ARCHEOPS point-sources (red-filled). The warm colour excess in the ARCHEOPS distribution is due to HII regions.

massive than the stellar cores themselves and hence, the cloud has not been disturbed yet.

\subsection{Number counts}

In Sect. 3.4, we have seen that a power law exponent of $1.5 \pm$ 0.2 gives a fair representation of the integral number counts. The integrated flux of these clumps (see Eq. (3)) is thus dominated by the faint sources. Because the submillimetre emission is optically thin, we can directly transform the submillimeter flux $F_{v}$ into a total clump gas mass using the following formula:

$M_{\text {gas }}=\frac{F_{v} D^{2}}{\kappa_{v} B_{v}(T)}=1.4 \times 10^{3} M_{\odot}\left(\frac{F_{v}(353 \mathrm{GHz})}{100 \mathrm{Jy}}\right)\left(\frac{D}{1 \mathrm{kpc}}\right)^{2}$,

where $B_{v}(T)$ is the Planck function and $D$ is the distance to the source. The last equality is obtained with the dust absorption coefficient $\kappa_{v}$ taken as $0.0012 \mathrm{~m}^{2} \mathrm{~kg}^{-1}$ (Preibisch et al. 1993) at $353 \mathrm{GHz}$ and an assumed average dust temperature $T$ of $14 \mathrm{~K}$.

Hence, for a distance range of $200 \mathrm{pc}$ to $2 \mathrm{kpc}$ the detected clumps have a mass range from 40 to $40000 M_{\odot}$, intermediate between cores and giant molecular clouds. The ARCHEOPS beam encompasses $3.5 \mathrm{pc}$ at a distance of $1 \mathrm{kpc}$, a "typical" intermediate size too. The likely dispersion in the distance of the different clumps prevents an interpretation of the flux number counts in terms of mass distribution and favors approaching an Euclidian count with an exponent of 1.5. For example, clumps close to Cygnus are likely at a distance of $1.7 \mathrm{kpc}$ (Schneider et al. 2006). Other clumps close to Taurus and Perseus associations are typically at a distance of about $200 \pm 60$ pc (Dame et al. 2001). Optical depth effects are not likely to play a major role in the fitting of Eq. (1) because a typical mass of $1000 M_{\odot}$ extending over 1 pc has an opacity of 0.01 only at $545 \mathrm{GHz}$ (using $\tau=4 \kappa_{v} M_{\text {gas }} /\left(\pi D^{2}\right)$ ).

The satellite PLANCK (The Planck collaboration 2005) with a thousand-fold increase in sensitivity should supersede the present results. In the mean time, the number counts allow us to firmly establish that there will be enough bright point-sources to reconstruct and monitor the PLANCK HFI (Lamarre et al. 2003) focal plane geometry. Indeed, each detector has an instantaneous sensitivity between 1 and 2 Jy per acquired sample (about $5 \mathrm{~ms}$ of integration). The sources above $100 \mathrm{Jy}$ will thus be detected with an excellent signal to noise ratio at each crossing. As the scans will drift by typically one degree per day, hundreds of sources will be available for accurate astrometry of the HFI detectors during the course of the PLANCK survey, roughly one every day, and probably more when crossing the inner Galaxy.

Moreover, a deep unbiased all-sky survey of submillimetre clumps will be available at the end of the PLANCK survey. With a final HFI sensitivity of about $10-50 \mathrm{mJy}(1 \sigma)$, by extrapolating ARCHEOPS number counts to the whole galaxy and to fainter fluxes, one can expect PLANCK to detect tens of thousands of submillimetre sources, actually down to the confusion limit, in the Galactic Plane.

\section{Conclusions}

This is a systematic extraction of the point sources from the ARCHEOPS last flight data. These point sources are valuable for the number counts of galactic sources in the (sub)millimetre domain and the study of the early star formation processes. The mean integration time per source is only of few tenths of seconds. Nevertheless, this shallow but wide survey has uncovered many new members of the family of very cold galactic clumps.

In general the ARCHEOPS point-sources can be associated with sources in the IRAS point-source catalogue or the IRAS small extended source catalogue. Only 30 ARCHEOPS pointsources out of a total of 304 remain unidentified.

The spectral energy distribution of the ARCHEOPS pointsources is compatible with a modified black body having as parameters the temperature $T$ and dust spectral index $\beta$. By fitting the spectrum of the ARCHEOPS sources to such a model we find 

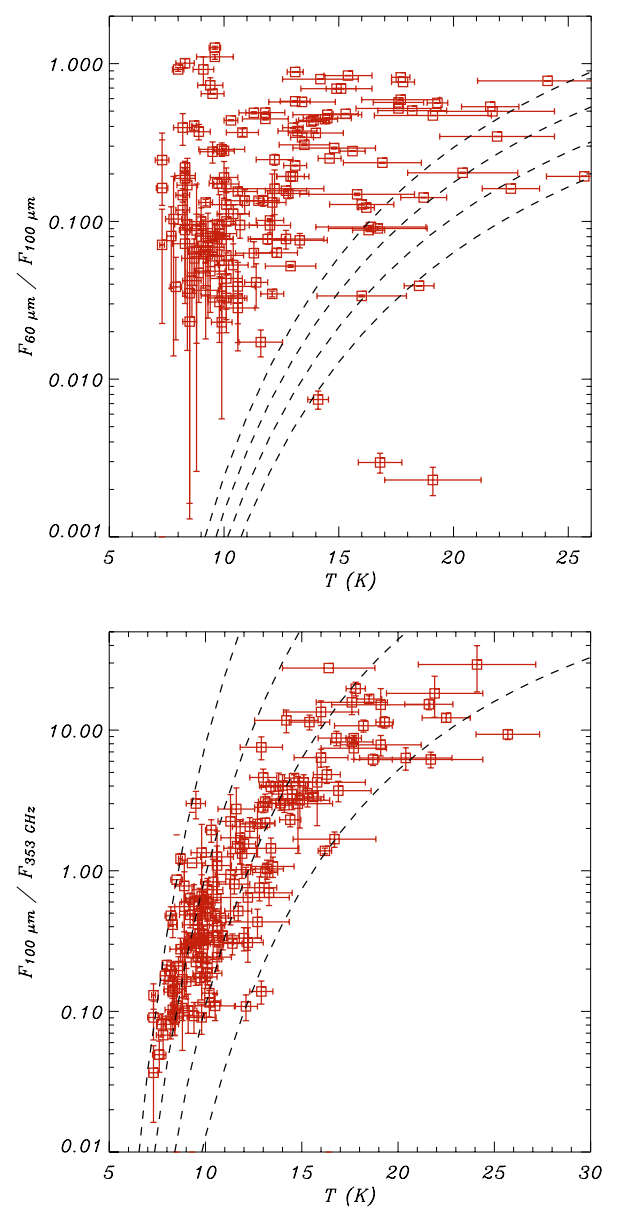

Fig. 14. Upper plot: far infrared IRAS colour as a function of $T$ for the ARCHEOPS point sources detected at 60 and $100 \mu \mathrm{m}$. Dashed lines in the two plots correspond to single temperature component models with an emissivity index of 1-4, from bottom to top. The two points at the lower right of the lines are due to an incorrect association with a $60 \mu \mathrm{m}$ source. Lower plot: submillimetre colour ( $100 \mu \mathrm{m}$ to $353 \mathrm{GHz}$ flux ratio) of ARCHEOPS point sources with a detection at $100 \mu \mathrm{m}$, as a function of $T$ for an acceptable fit of the spectral energy distribution.

that most ARCHEOPS point-sources are cold clumps with temperatures in the range of 7 to $27 \mathrm{~K}$. We also prove that there exists an inverse relationship between $T$ and $\beta$ : $\beta$ increases significantly with decreasing $T$.

We have found that most of the 302 ARCHEOPS pointsources have standard galactic IRAS far infrared colours, except for 40 identified as ultra compact HII regions, and 26 identified with other HII regions. Most of the clumps do not seem to be disturbed yet by internal sources. The far infrared colours are uncorrelated with the temperature deduced from the submillimetre fit. Only $\sim 1.3 \%$ of the IRAS sources at low galactic latitudes $\left(|b| \leq 10^{\circ}\right)$ have a bright submillimetre counterpart $\left(F_{v}(353 \mathrm{GHz}) \geq 50 \mathrm{Jy}\right)$. This means that it is difficult to predict which IRAS source will be detected in submillimetre surveys, for example, in PLANCK simulation of foreground to the CMB anisotropies.

Acknowledgements. The HEALPix package has been used throughout the data analysis (Gorski et al. 2005). This research has made use of the VizieR catalogue access tool, CDS, Strasbourg, France. We thank the ARCHEOPS collaboration for their efforts throughout the long campaigns. This work has been done with the PLANCK HFI data processing facility. We thank Bruno Bezard and Raphaël Moreno for very fruitful discussions about planets.

\section{References}

Baars, J. W. M., Genzel, R., Pauliny-Toth, I. I. K., et al. 1977, A\&A, 61, 99 Beichman, C. A., Neugebauer, G., Habing, H. J., Clegg, P. E., \& Chester, T. J. 1988, Infrared astronomical satellite (IRAS) catalogs and atlases, Explanatory supplement, 1

Benoît, A., Ade, P., Amblard, A., et al. 2002, Astropart. Phys., 17, 101

Benoît, A., Ade, P., Amblard, A., et al. 2003a, A\&A, 399, L19

Benoît, A., Ade, P., Amblard, A., et al. 2003b, A\&A, 399, L25

Benoît, A., Ade, P., Amblard, A., et al. 2004, A\&A, 424, 571

Bernard, J. P. 2004, 35th COSPAR Scientific Assembly, 4558

Boudet, N., Mutschke, H., Nayral, C., et al. 2005, ApJ, 633, 272

Chini, R., Kreysa, E., Mezger, P. G., \& Gemünd, H.-P. 1984, A\&A, 135, L14

Chini, R., Kreysa, E., Mezger, P. G., \& Gemünd, H.-P. 1986a, A\&A, 154, L8

Chini, R., Kreysa, E., Mezger, P. G., \& Gemünd, H.-P. 1986b, A\&A, 157, L1

Dame, T. M., Hartmann, D., \& Thaddeus, P. 2001, ApJ, 547, 792

Dunne, L., Eales, S., Ivison, R., Morgan, H., \& Edmunds, M. 2003, Nature, 424, 285

Dupac, X., Bernard, J.-P., Boudet, N., et al. 2003,A\&A, 404, L11

Dwek, E. 2004, ApJ, 607, 848

Finkbeiner, D. P., Davis, M., \& Schlegel, D. J. 1999, ApJ, 524, 867

Goldin, A. B., Kowitt, M. S., Cheng, E. S., et al. 1997, ApJ, 488, L161

Gomez, H. L., Dunne, L., Eales, S. A., Gomez, E. L., \& Edmunds, M. G. 2005, MNRAS, 361, 1012

Górski, K. M., Hivon, E., Banday, A. J., et al. 2005, ApJ, 622, 759 (http://healpix.jpl.nasa.gov)

Green, D. A. 2004, Bull. Astr. Soc. India, 32, 335, updates at (http://www .mrao.cam.ac.uk/surveys/snrs)

Hernández-Monteagudo, C., Macías-Pérez, J. F., Tristram, M., \& Désert, F.-X. 2006, A\&A, 449, 41

Hinshaw, G., Nolta, M. R., Bennett, C. L., et al. 2007, ApJS, 170, 288

Kurtz, S., Churchwell, E., \& Wood, D. O. S. 1994, ApJ, 91, 659

Lagache, G., Abergel, A., Boulanger, F., \& Puget, J.-L. 1998, A\&A, 333, 709L

Lamarre, J.-M., Puget, J.-L., Piat, M., et al. 2003, in Proceedings of the SPIE, IR Space Telescopes and Instruments, ed. J. C. Mather, 4850, 730

Macías-Pérez, J., Lagache, G., Maffei, B., et al. 2007, A\&A, 467, 1313

Macías-Pérez, J. F., Mayet, F., Désert, F.-X., \& Aumont, J. 2008a, A\&A, submitted

Macias-Perez, J. F., Mayet, F., \& Désert, F.-X. 2008b, in preparation

Miville-Dechênes, M.-A., \& Lagache, G. 2005, ApJS, 157, 302

Meny, C., Gromov, V., Boudet, N., et al. 2007, A\&A, 468, 171

Moreno, R. 1998, Ph.D. Thesis, Université Paris VI

Pajot, F., Stepnik, B., Lamarre, J.-M., et al. 2006, A\&A, 447, 769

Page, L., Barnes, C., Hinshaw, G., et al. 2003, ApJS, 148, 39

Paladini, R., Burigana, C., Davies, R. D., et al. 2003, A\&A, 397, 213

The Planck collaboration 2005, available at:

http://www.rssd.esa.int/SA/PLANCK/docs/

Bluebook-ESA-SCI (2005)1_V2.pdf

Ponthieu, N., Macías-Pérez, J. M., Tristram, M., et al. 2005, A\&A, 444, 327

Preibisch, Th., Ossenkopf, V., Yorke, H. W., \& Henning, Th. 1993, A\&A, 279, 577

Schneider, N., Bontemps, S., Simon, R., et al. 2006, A\&A, 458, 855

Tristram, M., Patanchon, G., Macías-Pérez, J. F., et al. 2005, A\&A, 436, 785 